\title{
Analysis for Topological Properties of the Network Feeding Usenet News
}

\author{
Yutaka Nakano \\ Graduate School of Informatics, Kyoto University \\ Kyoto 606-8501 Japan \\ nyutaka@net.ist.i.kyoto-u.ac.jp \\ Motonori Nakamura, Yasuo Okabe \\ Academic Center for Computing and Media Studies, Kyoto University \\ \{motonori,okabe\}@media.kyoto-u.ac.jp
}

\begin{abstract}
Recently, many studies have reported that various kinds of real networks, such as WWW, the Internet, metabolic system, BtoB transactions and so on, have the scale-free properties in common. We focus on the network feeding Usenet news where a node represents a news server and a link between two nodes represents a connection on which the two news servers exchane their articles. First we generate the whole topology map approximately from path information of a number of Usenet news articles. Then we examine topological properties of the network; degree distribution, degree correlation, average distance, clustering coefficient and community structures. We also compare these properties with those of theoretical network models. The analysis shows that there strongly exist a scale-free and a smallworld properties in the Usenet network. We also examine the community structure of this network and show that a small number of Usenet news servers that are highly contributive for article feeds forms the largest community and other servers tend to be categorized by their geographical locations.
\end{abstract}

\section{Introduction}

There in the world are a number of complex systems which can be regarded as large networks composed of nodes and links, such as the Internet, the World Wide Web (WWW), social relations, relations among genes, the metabolic system in a life body, a food web and business relations between companies. At the end of the 1990s, researches on common topological properties of such networks experienced a breakthrough with the seminal paper by Watts and Strogatz [46], which showed that these networks have the same properties in common: the small- world and the scale-free properties. The networks retaining these properties have a power-law degree distribution, a short distance between two given nodes and a high clustering coefficient, which can not be explained by the random graph theory mainly used for analysis of real networks so far. Subsequent works are so many and not a few on the Internet. The WWW, which is a network of Web pages, linked together by hyperlinks from one page to another, has been very heavily studied. Works by Albert et al. [7, 4], Kleinberg et al. [31], and Broder et al. [11] are particularly influential. The WWW also appears to have power-law in- and out-degree distributions, as well as a variety of other interesting properties $[4,31,11]$. Aiello et al. [1] have analyzed a network of telephone calls made over the AT\&T longdistance network on a single day. E-mail networks have also been studied by Ebel et al. [20], by Newman et al. [36], and by Guimer'a et al. [26]. Smith [43] reported similar results for instant messaging system. Studies of the structure of the Internet itself have been carried out by Faloutsos et al. [41, 22], Broida and Claffy [12], Chen et al. [16] and Chang et al. [14, 15].

The main subject of this paper is the Usenet News system (Netnews). The Netnews, in which millions of users discuss on tens of thousands of newsgroups, was established in 1980 and it had been used as the most common service on the Internet before the WWW was introduced. So far, there has been a number of researches on Netnews from a technical viewpoint; protocols, server performance studies and etc [29, 39, 42]. However, the Netnews has been paid very little attention by researchers in the area of complex networks except for a small portion of studies [9].

In this paper, we first generate the whole topology map approximately from path information in a number of Usenet news articles. Then we examine topological properties of the network; degree distribution, degree correlation, average distance, clustering coefficient and community struc- 
tures. We also compare these properties with those of theoretical network models.

The analysis shows that there strongly exist a scale-free and a small-world properties in the Netnews network. We also examine the community structure of this network and show that a small number of Netnews news servers that are highly contributive for article feeds form the largest community and other servers tend to be categorized by their geographical locations.

The rest of this paper is structured as follows. In Section 2, we present the definitions of topological properties. In Section 3, we introduce some theoretical network models. In Section 4, we first give the description as to what the Netnews is. Consequently, we show how we can examine our targeted network: the way of collecting topological information, the method to generate topology map. In Section 5, we present the result of our examination for the network and analyze its topological properties. In Section 6, we conclude our work and discuss future directions.

\section{Topological Metrics}

\subsection{Degree Distribution}

The two most basic network properties are the number of nodes $N$ and the number of links $E$. They define the average node degree as $\bar{k} \equiv \frac{2 E}{N}$.

Let $N_{k}$ be the number of nodes of degree $k$ ( $k$-degree nodes). Degree distribution is the probability that a randomly selected node has $k$-degree: $p_{k} \equiv \frac{N_{k}}{N}$. An way of presenting degree distribution is to make a plot of the cumulative distribution function: $P(k) \equiv \sum_{k^{\prime}=k}^{\infty} p_{k^{\prime}}$, which is the probability that the degree is greater than or equal to $k$. The cumulative distribution function of node degrees is referred to as CDF for node degrees in what follows. On the other hand, when we like to mention to the noncumulative degree distribution $p_{k}$, we refer to it as PDF (probability density function) for node degrees hereafter.

The PDF for node degrees contains more information about connectivity in a given network than the average degree, since given a specific form of $p_{k}$ we can always restore the average degree by $\bar{k}=\sum_{k=1}^{k_{\max }} k p_{k}$ where $k_{\max }$ is the maximum node degree in the network.

The Joint Degree Distribution (referred to as JDD) fills this gap by providing information about nodes' 1hop neighborhoods. Let $e_{k_{0} k_{1}}$ be the total number of edges connecting nodes of degrees $k_{0}$ and $k_{1}$. The JDD is the probability that a randomly selected edge connects $k_{0^{-}}$and $k_{1}$-degree nodes: $P\left(k_{0}, k_{1}\right) \equiv \frac{e_{k_{0} k_{1}}}{2 E}$, where the function $P\left(k_{0}, k_{1}\right)$ satisfies $P\left(k_{0}, k_{1}\right)=P\left(k_{1}, k_{0}\right)$ and $\sum_{k_{0}, k_{1}} P\left(k_{0}, k_{1}\right)=1$.

Note that $P\left(k_{0}, k_{1}\right)$ is different from the conditional probability $P\left(k_{1} \mid k_{0}\right)=\frac{\bar{k}}{k_{0}} \frac{P\left(k_{0}, k_{1}\right)}{p_{k_{0}}}$ that a given $k_{0}$-degree node is connected to a $k_{1}$-degree node. The JDD contains more information about the connectivity in a network than the degree distribution, since given a specific form of $P\left(k_{0}, k_{1}\right)$ we can always restore both the average degree $\bar{k}$ and the PDF for node degrees $p_{k}$ by these expressions:

$$
\bar{k}=\left(\sum_{k, k^{\prime}} \frac{P\left(k, k^{\prime}\right)}{k}\right)^{-1}, p_{k}=\frac{\bar{k}}{k} \sum_{k^{\prime}} P\left(k, k^{\prime}\right)
$$

The researchers studying real networks has recently started recognizing the importance on JDD [47].

The JDD is a function of two arguments. Let us define a summary statistic of JDD, that is a function of one argument indicating average degree of the nearest neighbor of a $k$-degree node: $k_{\mathrm{nn}}(k) \equiv \frac{\bar{k}}{k p_{k}} \sum_{k^{\prime}=1}^{k_{\max }} k^{\prime} P\left(k^{\prime}, k\right)$. It is simply the average neighbor degree of the average $k$-degree node. It shows whether nodes of a given degree preferentially connect to high- or low-degree nodes. In a full mesh network, $k_{\mathrm{nn}}(k)$ reaches its maximal possible value $N-1$. For uniform network comparison, we use normalized values $\frac{k_{\mathrm{nn}}(k)}{N-1}$.

We can further summarize the JDD by a single scalar called assortativity coefficient $r$ (or called the Pearson correlation coefficient [34]):

$$
r \equiv \frac{\bar{k} \sum_{k} k^{2} k_{\mathrm{nn}}(k) p_{k}-\left\langle k^{2}\right\rangle^{2}}{\bar{k}\left\langle k^{3}\right\rangle-\left\langle k^{2}\right\rangle^{2}}
$$

where $\left\langle k^{n}\right\rangle=\sum_{k, k^{\prime}} k^{n-1} P\left(k, k^{\prime}\right)$, that is, the $n$-th mean of $P\left(k_{0}, k_{1}\right)$. $r$ satisfies $-1 \leq r \leq 1$.

\subsection{Clustering Coefficient}

While the JDD contains information about the degrees of neighbors for the average $k$-degree node, it does not tell us how these neighbors interconnect. Clustering coefficients satisfies this need by providing a measure of how close a node's neighbors are to forming a clique. In the language of social networks, it can be described that how the friend of your friend is likely also to be your friend. In terms of network topology, high clustering coefficient means the presence of a heightened number of triangles in the network, i.e., sets of three nodes each of which is connected to each of the others. It can be quantified by defining clustering coefficient $C$ as

$$
C \equiv \frac{3 \times \text { number of triangles in the network }}{\text { number of pairs of adjacent links }}
$$

where "number of pairs of adjacent links" can be transcribed that "number of sets of three nodes each of which is connected to each of others".

To characterize how the network is clustered around each node, let us give an definition of local clustering $C(k)$ as a function of node degree $k$. Let $\bar{m}_{\mathrm{nn}}(k)$ be the average number of links between the neighbors of $k$-degree nodes. 
Clustering coefficient is the ratio of this number to the maximum possible such links: $C(k) \equiv \frac{2 \bar{m}_{\mathrm{nn}}(k)}{k(k-1)}(k \neq 0,1)$. If the degree $k$ equals 0 or 1 , we define $C(k)=0$.

If two neighbors of a node are connected, then these three nodes together form a triangle. Therefore, by definition, local clustering is the average number of triangles involving $k$-degree nodes.

\subsection{Distance}

Let $N_{d}(x)$ be the number of pairs of nodes to be a distance $x$ hops from each other. The distance distribution $d(x)$ is the PDF for a random pair of nodes to be at a distance $x$ hops from each other, defined as $d(x) \equiv \frac{2 N_{d}(x)}{N(N-1)}$.

Let us define $\bar{d}$ to be the mean shortest distance between node pairs in a network: $\bar{d} \equiv \frac{2}{N(N-1)} \sum_{i \leq j} d_{i j}$, where $i$ and $j$ are the index number of two nodes in a network and $d_{i j}$ is the shortest distance from node $i$ to node $j . \bar{d}$ determines the effective "linear size" of a network, the average separation of pairs of nodes [19]. For a lattice of dimension $\delta$ containing $N$ nodes, obviously, $\bar{d} \sim N^{1 / \delta}$. In a fully connected network, $\bar{d}=1$. One may roughly estimate $\bar{d}$ of a network in which random nodes are connected. If the average number of nearest neighbors of a node is $z_{1}$, then about $z_{1}^{l}$ nodes of the network are at a distance $l$ from the nodes or closer. Hence, $N \sim z_{1}^{\bar{d}}$ and then $\bar{d} \sim \ln (N) / \ln \left(z_{1}\right)$, i.e., average shortest distance value is small even for very large random graph. In our work, we attempt to infer the presence of "small-world effect" using the metric $\bar{d}$.

\subsection{Community Structure}

As some researchers pointed out [24, 40, 30, 10], a property that seems to be common to many networks is community structure, the grouping of network nodes into subgraphs within which the network connections are dense, but between which they are sparser. To find and analyze such groups can provide invaluable help in understanding and visualizing the structure of networks.

In this paper, we analyze the community structure of news servers, using Newman's method [35]. He showed that this method has a considerable speed advantage over previous methods $[40,24,30,10,37]$, running to completion in time that scales $O((E+N) N)$, or $O\left(N^{2}\right)$ for a sparse graph in the worst case (where $N$ and $E$ express the number of nodes and links, respectively).

\section{Network Models}

\subsection{Connecting Nearest Neighbor Model}

The connecting nearest neighbor model (CNN-model) is a theoretical model proposed by Alexi [45]. It generates a network with scale-free property and also high clustering coefficients. In this model, links are tied by the activities of nodes, which are different from the preferential attachment of Barabàsi and Albert's model (BA-model)[3], as he intended this model for the simulation of social networks. Thus, we considered that the CNN-model is suitable to find the differences between the feed network and the network constructed only by local interactions between nodes. The process of network generation probabilistically proceeds as follows:

(i) With probability $(1-u)$, one new node is introduced in the network, one new link created at the same time and it connect the new node with node $j$ selected at random. Plus, the potential links from the new node to all the neighbor of $j$ are created. Note that at this time, the potential links are not appeared in the network.

(ii) With probability $u$, one potential link selected at random is converted into an existing link.

He showed the power-law degree distribution (in PDF) of this model, $p_{k} \sim k^{-\gamma(u)}$ where $\gamma(u)$ has the form

$$
\gamma(u)=1+\frac{2(1-u)}{u}\left(-1+\sqrt{1+\frac{4(1-u)}{u}}\right)^{-1},
$$

with the limiting cases $\gamma(0)=\infty, \gamma(1)=2$. The author obtained the rough results of the clustering coefficient $C(k)$ and the nearest neighbor connectivity $k_{\mathrm{nn}}(k)$ of this model (where $k$ expresses a node degree). Firstly, clustering coefficient results in $C(k) \sim k^{-\beta}$ for some exponent $\beta$, where $\beta$ satisfies $0<\beta<1$. Secondly, the nearest neighbor connectivity results in $k_{\mathrm{nn}}(k) \sim k^{\alpha}$ for positive constant $\alpha$. What we should notice here is $C(k)$ exhibits a power-law distribution. On the contrary $k_{\mathrm{nn}}(k)$ follows a power function with positive exponent.

\subsection{Configuration Model}

The configuration model produces a maximally randomized graph with a given degree distribution (PDF) $p_{k}$. Rewiring the links in the original network with degree distribution $p_{k}$ randomly, this model generates a new network having strictly the same degree distribution as original one.

When the random nature of the link assignment is given, this algorithm generates networks with the expected degree distribution, and the noticeable point is that generated networks have no correlations for node degree [13], i.e., Configuration-model generates networks with assortativity coefficient $r=0$ in theory. The authors of [19] showed that an average neighbor connectivity $k_{\mathrm{nn}}(k)$ of Configuration-model is a constant function of node degree, $k_{\mathrm{nn}}(k)=\frac{\left\langle k^{2}\right\rangle}{\bar{k}}$, where $\left\langle k^{2}\right\rangle$ is defined by equation $\left\langle k^{n}\right\rangle=\sum_{k, k^{\prime}} k^{n-1} P\left(k, k^{\prime}\right)$.

This feature of Configuration-model is very useful for practical purpose because it enables us to test a presence or 
an absence of degree correlation in real networks. Another feature is that the local clustering is also a constant function of node degree, $C(k)=\frac{\left\langle k^{2}\right\rangle-\bar{k}^{2}}{N \bar{k}^{3}}$, where $N$ is the number of nodes.

\section{Netnews and Topology Construction}

\subsection{The Usenet News}

Along with email, the Usenet News (or Netnews in short) is one of the oldest communication methods on the Internet and its predecessors, since when two graduate students of Duke University, Tom Truscott and Jim Ellis, thought of hooking computers together to exchange information within the UNIX community in late 1979.

The Netnews can be considered as a large hierarchical set of bulletin board system concerning various topics. The Netnews are categorized according to interest and the categories are called "newsgroups". The name of the newsgroup provides the hierarchical category information. The most famous top eight categories of newsgroups run by numerous news servers all around the world, i.e. "comp.", "humanities.”, “misc.”, “news.”, “rec.”, “sci.”, “soc.”, “talk.”, are referred as "BIG8."

Newsgroups are decentralized, which means that the articles are not stored on a single server, but are replicated to thousands of servers around the world. Each user downloads articles from or posts articles to a (possibly nearest) Netnews server, and a large number of servers distributed on the Internet store and forward articles to neighbouring servers.

At first, the news articles are exchanged by the Unix to Unix CoPy (UUCP) [27]. In 1984, the Network News Transfer Protocol (NNTP) [29] was developed so that it can enable news servers to exchange news articles more efficiently with the standard TCP/IP protocol.

NNTP defines some essential headers: "From", "Date", "Newsgroup", "Subject", "Message-ID", "Path". Fig. 1 shows an example of the header of a news article in a news server. The "Path" line shows the route the article took to reach the server. It is shown as a sequence of server names. When a server forwards a message, it adds its own name at the beginning of server list in the path line. The names are separated by a punctuation character. Normally, the rightmost name will be the name of the originating system, but it is also permissible to include an extra entry on the right, like the name of the sender. The main use of information in a path line is to cut down on redundant network traffic by omitting to forward articles to neighbouring servers that are known to have the articles already.

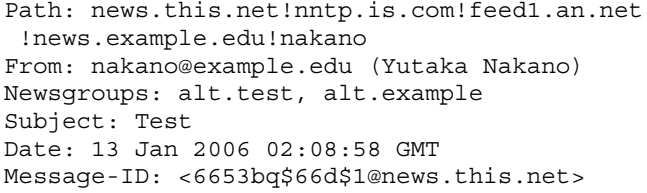

\section{Figure 1. NNTP header of an article}

\subsection{Methodology for Topology Inference}

\subsubsection{Topology Inference Using Path Information}

The process of generating a topology map of the feed network starts with obtaining "Path:" lines in headers of news articles from news servers as many as possible. As described before, a path line shows the route the article traveled to reach the news server which stores the article. Consider the case where we collect articles from a server news.this.net (source server) and one of the obtained article has the path line same as the example shown in Fig. 1. That path line indicates that the article posted by nakano passed through servers news.example.com, feed1.an.net, nntp.is.com, news.this.net, in that order. We can infer that there are links between news.example.com and feed1.an.net, between feed1.an.net and nntp.is.com, and between nntp.is.com and news.this.net.

Although we can get a presumably accurate topology by checking path lines, there can be still lack of nodes and links due to the insufficiency of information. To be specific, if path information is obtained only from a single news server, or if the amount of articles are quite small, the method mentioned above may amount to the collection of shortest paths from that news server and we may fail to find minor/redundant links and servers far from the source server [17]. Therefore, to avoid this insufficiency of information, we should collect enough amount of various path information in in from multiple servers that are not next to each other but enough away from each other.

\subsubsection{Data Sources}

Table 1 shows the list of source news servers from which we obtained the path information. We chosed 36 servers from ones opened for free and collected path information from articles on the listed servers. In our work, we selected the specific newsgroups like BIG8 or "alt." as the sources of articles, since articles in that news groups are seems to be distributed around the world. We believe that the property of a worldwide network should be distinguished from the property which a localized network holds - there may be some servers which feed only localized newsgroups like "fj." or "jp.". Therefore, we ignored such localized newsgroups so that we don't mix up the properties of a worldwide network 
with the one of a localize network. Moreover, we selected articles posted within the 2005 because old articles may give us obsolete path information, i.e., there can be a name of a news server already stopped working. We collected path information from Sept. 2005 to Dec. 2005 and the total quantity of obtained articles amounted to 6,704,210. The amount of articles obtained from each server is shown on the right side of server names in Table 1.

\section{Table 1. List of source servers and quantity of obtained articles}

\begin{tabular}{lr|lr} 
Server Name & Qty of articles & Server Name & Qty of articles \\
\hline \hline news.media.kyoto-u.ac.jp & 1740709 & news.mtu.ru & 57569 \\
msnews.microsoft.com & 1046283 & mx.iis.nsk.su & 44812 \\
news.edisontel.com & 922496 & news.pyrenet.fr & 40918 \\
freenews.netfront.net & 418223 & news.aioe.org & 35361 \\
news.amu.eedu.pl & 314594 & secrews.netscape.com & 22831 \\
news.srv.cquest.utront.ca & 251702 & deer.math.nctu.edu.tw & 15026 \\
forums.novell.com & 228279 & news.stack.nl & 11771 \\
news-east.usenet.com & 213975 & nan.host-web.net & 8377 \\
news.c99.net & 202385 & news.in-ulm.de & 5901 \\
netnews.eranet.net & 182145 & news.flora.ca & 5718 \\
news.f.de.plusline.net & 177431 & acc.sim.net.ua & 5140 \\
news.isu.edu.tw & 149484 & news.vsi.ru & 2644 \\
fuchur.bw-networx.net & 142070 & serv3.vsi.ru & 2352 \\
nntp.idg.pl & 137405 & pubnews.gradwell.net & 2042 \\
news.readfreenews.net & 90784 & dizmila.cavern.pl & 1755 \\
news2.mneel.com & 82651 & news.hs-neiderrhein.de & 252 \\
dp-news.maxwell.syr.edu & 78250 & ns.musin.de & 187 \\
humbolt.leper.phil.uu.nl & 62509 & sirius.sns.net.ua & 179 \\
\hline \hline \multicolumn{3}{c}{}
\end{tabular}

\subsubsection{Canonicalizing Path Information}

Although the data sets obtained from the "Path:" line of articles represent the current state of the news-feeding network, they are inexact and indirect reflections of the underlying topology. They require preprocessing before generating the desired topology map. For all our data sets, we must make choices while dealing with ambiguities in the raw data. In this section, we describe the canonicalizing process to make our data sets close to the real topology. By the NNTP regulation, the header of a news article is rewritable, which means that every news server can freely change the string after the "Path:" in header of the received article. Then, the path information obtained from news servers may include extra entries which don't correspond to real news servers.

Firstly, let us take a typical example in (a) of Fig. 2. The strings netnews and not-for-mail, which are located at the second from the end and at the end of path line, were automatically added by user's program which is used for posting to indicate that the path information can not be used as (UUCP style) email addressing. Hence, these extra entries need to be ignored when we interpret this path line.

Consider the case in (b) of Fig. 2, a string .POSTED is appended to the existing server name news.ono.com, which means that the article was posted to news.ono.com at first; the string news.ono.com.POSTED should be changed to news.ono.com.

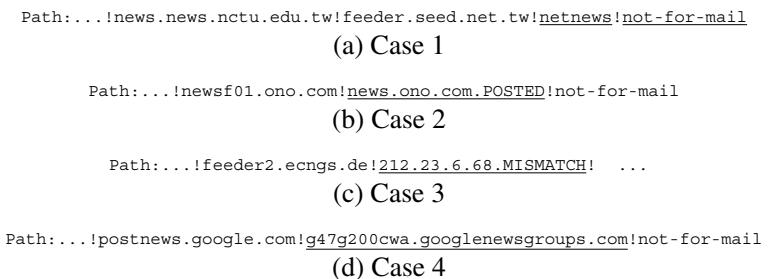

Figure 2. Path information to be filtered

The third case shown in (c) of Fig.2 is a little more complicated. There may be a entry 212.23.6.68.MISМАТСH, where 212.23.6.68 indicates the IP-address of a news server. The rest of the string .MISMATCH means that the adjacent server feeder2.ecngs.de found some inconsistency between configuration and information in the article from the server 212.23.6.68. In this case, we should rewrite 212.23.6.68.MISMATCH to its FQDN, hamilton.zen.co.uk.

These examples shown are, in fact, not difficult to handle as they can be systematically transcribed by means of simple string manipulation. However, there are much more entries difficult to decide whether to be removed or not. For instance, consider the path line shown in (d) of Fig. 2. The entry g47g2000cwa.googlegroups.com looks like a FQDN of an existing server but actually it is mere a "stamp" indicating that the article was posted in the "google groups" which is one of the service provided by Google. We faced a large number of entries similar to this case during the canonicalizing process of path information. Although such entries are difficult to process automatically, to leave them lay may cause crucial errors in the statistical properties of resulting topology. Therefore, we checked all entries appeared in the path lines and manually built the "black list" where the wrong entries are listed. The listed wrong entries amounted to 11,965 .

All path information obtained from collected articles were once canonicalized through the rewrite process described in the above three cases and cleaned once again by means of the "black list". After the preprocessing, the topology map for further analysis is generated from the path information. Note that number of articles forwarded on links does not matter to generate the topology map.

\section{Topological Properties}

In this section, we present our observed results, and perform not only the analysis of the results of the feed network but also the comparison with the topological properties of network models.

For simplicity, we will refer to the network obtained by the path information collected from servers listed in Table 1 
as NEWS. As the most basic result, the network size and the number of links of NEWS results in 6,152 and 25,130, respectively (referred to as $N_{\text {NEWS }}$ and $E_{\text {NEWS }}$ in the rest of this paper).

\subsection{Node Degree}

We first show the results about node degrees. The average node degree is 8.17 while the maximum node degree is 329. The node degree distribution in CDF of NEWS is shown in Fig. 3. The CDF exhibits a monotonically decreasing distribution in log-log scale. Especially, the correlation coefficient is greater than $99 \%$ in the range from degree 2 to around 30 (the range $\mathbf{A}$ ) where the $66 \%$ of nodes belong, meaning that the CDF has strong linearity in that range. We found that the slope in the range $\mathbf{A}$ is fitted to -0.87 which means that the CDF of NEWS is proportional to $k^{-0.87}$ where $k$ expresses the node degree. However, in the range from degree 30 to 329 (the range $\mathbf{B}$ ), its linearity breaks and decays faster than the expected power-law distribution $k^{-0.87}$ drawn in the dashed line. The CDF for node degree of NEWS follows power-law in the range $\mathbf{A}$ but it deviates from strict power-law in the range $\mathbf{B}$, which means that the scale-free property is limited in the particular range of degree.

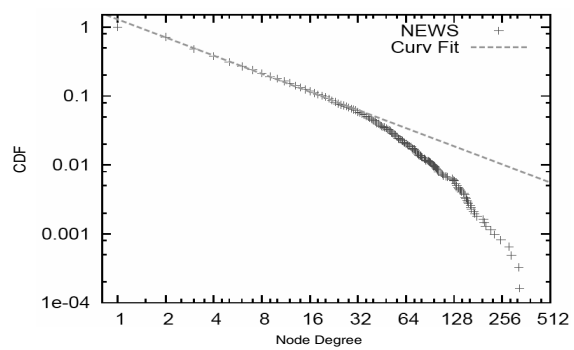

Figure 3. The CDF of NEWS

The differences between the real topology and the theoretical model are considered to be caused by several administrative restrictions for running a news server. The topology of NEWS may belong to a family of networks that obeys power-law up to a finite threshold and decays exponentially above this threshold, which is pointed out by some researches $[2,8]$.

\subsection{Degree Correlations}

In this section, we discuss the degree correlations of the feed network. For comparison, we consider a network generated by Configuration-model with the same degree distribution as that of NEWS (referred to as Conf hereafter). The number of nodes and links of Conf both equal to NEWS's, and Conf is generated in such a way
Table 2. Number of nodes in networks and assortativity coefficient $r$ for a number of different networks

\begin{tabular}{lccc}
\hline \hline Network & Number of Nodes & Number of Links & Assortativity $r$ \\
\hline Physics co-authorship (a) & 52909 & 245300 & 0.363 \\
Biology co-authorchip (a) & 1520251 & 11803064 & 0.127 \\
Mathematics co-authorship (b) & 253339 & 496489 & 0.120 \\
Film actor collaborations (c) & 449913 & 25516482 & 0.208 \\
Company directors (d) & 7673 & 55392 & 0.276 \\
\hline Internet (e) & 10697 & 31992 & -0.189 \\
WWW (f) & 269504 & 1497135 & -0.065 \\
peer-to-peer network(g) & 880 & 1296 & -0.366 \\
electronic circuits(h) & 24097 & 53248 & -0.154 \\
\hline Protein interactions (i) & 2115 & 2240 & -0.156 \\
Neural network (j) & 307 & 2359 & -0.163 \\
Marine food web (k) & 134 & 598 & -0.247 \\
Freshwater food web & 92 & 997 & -0.276 \\
\hline NEWS(l) & 6152 & 25130 & $\mathbf{0 . 0 0 1 1 9}$ \\
Conf(m) & 6152 & 25130 & -0.097 \\
CNN(n) & 6152 & 25130 & 0.216 \\
\hline Random Graph(o) & & & 0 \\
BA-model(p) & & & 0 \\
\hline \hline
\end{tabular}

that the degree distribution corresponds to NEWS. Another example for comparison is a network derived from CNN-model (referred to as $\mathbf{C N N}$ ) as well in which the author of [45] found that the nearest neighbor connectivity follows $k_{n n}(k) \sim k^{\alpha}$ for a constant $\alpha$. A comparison of the assortativity coefficient of NEWS with several networks is shown in Table 2. Here collaboration networks of (a) scientists in physics and biology [33], (b) mathematicians [25], (c) film actors [46], and (d) businesspeople [18]; (e) connections between autonomous systems on the Internet [16]; (f) directed hyperlinks between Web pages in a single domain [5]; (g) Gnutella network [38]; (h) electronic circuits [23]; (i) protein-protein interaction network in yeast [28]; (j) undirected (and unweighted) synaptic connections in the neural network of the nematode C. Elegans [46]; (k) undirected trophic relations in the food web of Little Rock Lake, Wisconsin [32]; (1) the feed network of Netnews (our obtained network); (m) the network generated by Configuration-model; (n) the network generated by CNN-model; (o) the random graph model of Erdös and Rényi [21] (theoretical value); (p) BA-model [6] (theoretical value). As we introduced in Section 2, the assortativity coefficient $r$ gives us direct implication about the network topology.

The assortativity coefficient $r$ of NEWS is 0.00119 , which is fairly lower than social networks shown in the table and CNN. The degree correlation in NEWS is far weaker than social networks. Interestingly, however, while two technological networks such as the Internet and the WWW have negative assortative coefficients, assortativity $r$ of NEWS, which is one kind of technological network, keeps positive value close to zero. We can characterize NEWS as a network close to uncorrelated network, i.e., the degrees at the end points of any link are independent. Furthermore, NEWS is not a disassortative network unlike the Internet and the WWW while it's much less assortative than 
social networks.

\subsection{Distance}

In this section we show the results about node distance of NEWS. We calculated some distance metrics with breadthfirst search. It allows us to calculate exhaustively the length of the shortest paths from every node on a network to every other in time $O(N E)$, where $\mathrm{N}$ and $\mathrm{E}$ expresses the number of nodes and links, respectively. We have done this for nodes in NEWS and averaged these distance to find the mean distance between any pair of news servers. The distance distribution of NEWS is shown in Fig. 4 where the probability for a random pair of nodes to be at a distance indicated in the horizontal axis from each other. As Fig. 4 shows, the distance between two nodes in NEWS follows a normal distribution which has the peak at distance 4 hops. The average distance is $\bar{d}_{N E W S}=4.38$. As this result shows, the distance between the nodes is all quite small compared to the network size. This result is considered to be the evidence supporting the "small-world" effect in the feeding network. This small-world effect is probably a good sign for article feed; it shows that articles will not have far to travel through the network to reach the end readers.

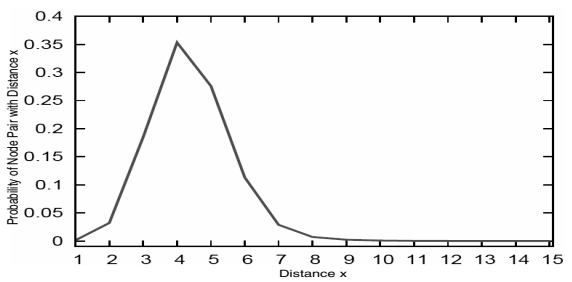

\section{Figure 4. The distance distribution $d(x)$ of NEWS}

\subsection{Clustering Coefficients}

Next we focus on how nodes are clustered in NEWS. Again, the clustering coefficient of the network reflects the "cliquishness" [19] of the mean closest neighborhood of a node, that is, the extent to which the nearest neighbors of a node are the nearest neighbors of each other. We show the local clustering of NEWS, Conf and CNN as a function of node degree in Fig. 5. The local clustering of NEWS shows weak decay with increasing of node degree.

We compare the clustering coefficients of each network of NEWS, Conf and CNN; 0.176, 0.0377 and 0.517, respectively. NEWS and Conf are still different as to their clustering coefficients although they share the same tendency in assortativity as examined in Section 5.2. NEWS

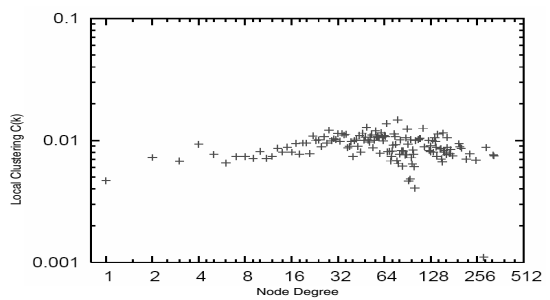
Figure 5 . The distributions of local clustering
$C(k)$ of NEWS

isn't constructed by local interactions between nodes like CNN because of the low local clustering in the area of lowdegree nodes as opposite to $\mathbf{C N N}$.

\subsection{Community Structure}

So far, we have reviewed the results regarding overall structural factors like degree distributions, clustering coefficients, average distance, etc. In this section, let us examine the community structure of the feed network to reveal more details on how the network is structured. We believe that visualization of networks is one of the most useful and intuitively comprehensible tool to understand the community structure. For the visualization, we used the software called "Otter" developed by Bradley Huffaker, Evi Nemeth and $\mathrm{K}$. Claffy in CAIDA. In our visualization, nodes included in the same community are put together into one disc-shaped area separated from other communities. Within a community, the higher the node degree becomes, the closer to the center of the area the node is located. In addition, the largest community is located on the center of the figure and the others are located around the largest one so as not to lap over each other.

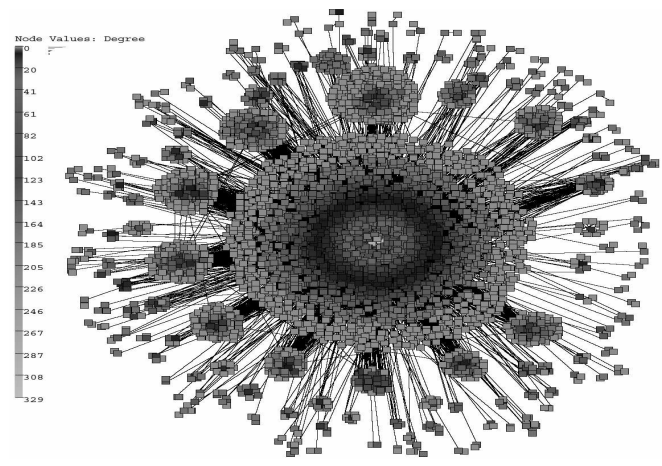

Figure 6. Visualization of NEWS.

The visualization of NEWS is shown in Fig. 6. Firstly, 
we can find an outstanding large community in the center of the picture. The largest community size (the number of nodes belonging to the community) is 3144 meaning that it includes over $50 \%$ of all nodes, while the smallest community size is 2. Consequently, NEWS is grouped into 139 communities and the number of communities of size 2 is 72. The largest community is surrounded by other communities whose sizes are all quite smaller than the largest one. Considering degree of nodes, there are a few nodes which have high node degree at the center of each groups, and the nodes are surrounded by many other nodes which have node degree lower than 20 . In other words, scale-free property appears in the visualization of NEWS. Another point captured from Fig. 6 is that high degree nodes aren't decentralized all over the communities but centered on the largest

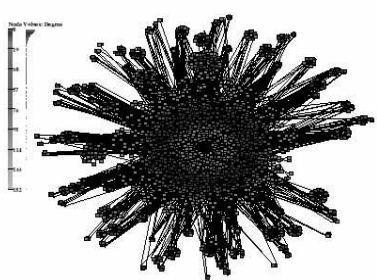

(a) $\mathbf{C N N}$

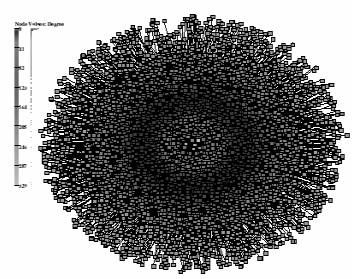

(b) Conf

\section{Figure 7. The visualization of $\mathrm{CNN}$ and Conf}

We have also performed the Newman's algorithm to Conf and CNN and the result is shown in Fig.7. The community structure of NEWS looks similar to the appearance of $\mathbf{C N N}$, and these are composed of many communities as opposed to Conf.

Next, we examine Fig. 6 from another point of view, that is, domains of news servers. Observing the largest community and the second largest community, they look like composing of servers having miscellaneous domain names, but there also exist some other communities. For instance, let us look closely at the third largest community. It mainly composed of .jp domain servers. The percentage of each domain name in this community is shown in (c) of Table 3. About $70 \%$ of nodes in this community belong to .jp domain.

The sixth largest community is mainly comprised of .pl domain servers, which takes over $62.3 \%$ of all the nodes in the community (for detailed percentage, see (d) of Table 3 ). We can find much more such communities consisting mostly of news servers having the same domain name, e.g., .uk, .edu and .de communities. Such grouping into communities may be made heavily depending on domain names of each news server. In other words, geographical locations of news servers strongly affect to the community
Table 3. The percentage of domain names of news servers

\begin{tabular}{l|cccccccc}
\multicolumn{8}{c}{ (a) The largest community } \\
\hline \hline Domain & .net & .com & .de & .nl & .edu & .org & .it & other \\
\hline Percentage & $\mathbf{3 1 . 5 \%}$ & $21.9 \%$ & $5.5 \%$ & $4.8 \%$ & $4.3 \%$ & $2.5 \%$ & $1.9 \%$ & $27.6 \%$ \\
\hline \hline \multicolumn{8}{|c}{ (b) The second largest } & community \\
\hline \hline Domain & .net & .com & .ca & .uk & .nl & .nz & .edu & other \\
\hline Percentage & $\mathbf{4 5 . 3 \%}$ & $40.0 \%$ & $4.6 \%$ & $2.7 \%$ & $2.1 \%$ & $1.0 \%$ & $0.7 \%$ & $3.6 \%$ \\
\hline \hline
\end{tabular}

(c) The third largest community

\begin{tabular}{l|ccccc}
\hline \hline Domain & .jp & .com & .net & .org & other \\
\hline Percentage & $\mathbf{6 8 . 5 \%}$ & $5.5 \%$ & $5.2 \%$ & $4.4 \%$ & $16.4 \%$ \\
\hline \hline \multicolumn{7}{c}{} \\
\multicolumn{7}{c}{ (d) The sixth largest community } \\
\hline \hline Domain & .pl & .ua & .net & .org & other \\
\hline Percentage & $\mathbf{6 2 . 3 \%}$ & $17.6 \%$ & $16.4 \%$ & $1.26 \%$ & $2.44 \%$ \\
\hline \hline
\end{tabular}

structure.

However, we are still not sure of how the largest community and the second largest one were made and why the largest community is so large. To clarify this, we have performed some more examinations. The percentage of domain names of news servers in these two communities respectively shown in (a) and (b) of Table 3 . As the two tables show, news servers included in .net and .com domain form a large part of each community, but this fact can not imply the critical reason of the grouping into such two groups. We examined the average distance of nodes included in the largest community and compared it with the average distance of nodes outside the community. The average distance of largest community results in 3.98 while the average of rest is 4.80 (cf. the average of all nodes is 4.38 ). The average distance of nodes in the largest community is smaller than the one of outside the largest community. This result gives us an one implication that the largest community is a "core" of the feed network: news articles mainly go through that community to shortcut and the servers included in the largest community play a vital role when feeding articles.

To assure our hypothesis, we investigated the relations between top1000.org [44] rankings of news servers and communities where the news servers are included. Top1000.org is a web site where thousands of news servers are ranked by contribution for article feed, which means that high-ranked news servers have relayed more articles than low-ranked servers. Table 4 shows news servers highranked in the ranking of Feb, 8th 2006 with their belonging communities. The bold number $\mathbf{n}$ in the second column represents that the server in the third column belongs to the $\mathbf{n}$-th largest community. News servers with N/A are the servers not found in our obtained path information. As Talbe 4 shows, except for three servers, all news servers belong to the largest community. About $30 \%$ of news servers ranked from top 1 to top $5000(1,445$ servers) are in the largest community. Obviously from these examinations, the 


\section{Table 4. List of high-ranked servers in top1000.org (from top 1 to 30 )}

\begin{tabular}{|c|c|c|}
\hline Top1000 rank & $\overline{\text { Community }}$ & 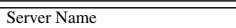 \\
\hline 1 & 1 & nntp.giganews.com \\
\hline 2 & 1 & news astraweb.com \\
\hline 3 & 1 & news.glorb.com \\
\hline 4 & 1 & feed.tweaknews.nl \\
\hline 5 & 1 & news.usenetserver.com \\
\hline 6 & 1 & newsrouter-eu.astraweb.com \\
\hline 7 & 1 & border2.nntp.ams.giganews.com \\
\hline 8 & 1 & border1.nntp.dca.giganews.com \\
\hline 9 & 1 & newshosting.com \\
\hline 10 & N/A & news.highwinds-media.com \\
\hline 11 & 1 & border2.nntp.dca.giganews.com \\
\hline 12 & 1 & feeder1.cambrium.nl \\
\hline 13 & 1 & proxad.net \\
\hline 14 & 1 & border1.nntp.ams.giganews.com \\
\hline 15 & 1 & npeer.de.kpn-eurorings.net \\
\hline 16 & 2 & local01.nntp.dca.giganews.com \\
\hline 17 & 1 & hwmedia \\
\hline 18 & 1 & news.tele.dk \\
\hline 19 & 1 & newsfeed.freenet.de \\
\hline 20 & 1 & lightspeed.eweka.nl \\
\hline 21 & 1 & feeder.xsnews.nl \\
\hline 22 & 48 & newsfeed.eweka.nl \\
\hline 23 & 1 & hwmnpeer01.ams \\
\hline 24 & 1 & easynews.com \\
\hline 25 & 1 & easynews \\
\hline 26 & 1 & nx02.iad01.newshosting.com \\
\hline 27 & 1 & novia \\
\hline 28 & 1 & news2.euro.net \\
\hline 29 & 1 & news.nctu.edu.tw \\
\hline 30 & 1 & postnews.google.com \\
\hline
\end{tabular}

largest community includes many of such high-ranked news servers. In other words, there may be many "contributive" servers in the largest community. This fact can be interpreted in this way: since an administrator who like to feed as many articles as possible preferentially makes connections with other news servers already highly contributive for article feed, connections between high-ranked servers increased, and hence, the community of high-ranked servers was created.

\section{Conclusion}

In this paper, we have studied networks feeding Usenet news articles where nodes represent news servers, and a link between two nodes represents a relationship of article feed between the two servers. Collecting path information from the multiple news servers, we have constructed the network which reflects the current topology of the feed network. For this network, we have calculated a number of statistics, comparing with some networks generated by theoretical network models.

As a consequence, the current feed network has a degree distribution which follows a "scale-free", but a scale-free property emerged only in the limited range (from degree 2 to 30) and the distribution deviates from a strict power-law outside that range. The Netnews network is closer to an "uncorrelated" network for node degree, i.e., the neighbors' degrees are independent of each node.

Another important feature of the network is "smallworld" property. We examined average distance of nodes in the feed network and it resulted in small values as a whole in comparison with the network size. The small average distance, which is result in about 4 hops, shows that articles posted at a news server will averagely pass through only three servers to go around the world. But we must also note that the situation might have been different in the UUCP age. We also examined the clustering coefficient of the feed network and local clustering of nodes. The feed network has higher value than Configuration-model but the value is fairly lower than $\mathrm{CNN}$-model.

Finally, we performed visualization of the network and reconfirmed the structural difference among theoretical network models. In particular, the community structure of the feed network is similar to the network derived from CNNmodel from the perspective of the number of communities and community size distribution.

We note that our work suffers from a number of methodological limitations and biases. Since our topology map is generated according to a number of Netnews articles, we may overlook existing news servers if there aren't enough articles available. In an extreme case, for example, if we can obtain only one path information, we will generate nothing but a topology where all nodes are aligned on a straight-line. In another case, if there's a news server extremely rarely used, it also may be difficult to find the articles came from that server, and hence, we probably miss that server. Although we consider such limitations as unavoidable, to validate topological properties of the feed network by means of statistics is still worth trying in the future work.

Acknowledgments The authors would like to express appreciation to Dr. Kenichi Okada of Tokyo Institute of Technology and Mr. Hiroshi Tsutsui of Kyoto University for their technical advises from a viewpoint of an administrator of a news server.

\section{References}

[1] W. Aiello, F. Chung, and L. Linyuan. A random graph model for massive graphs. in Proceedings of the 32nd Annual ACM Symposium on Theory of Computing, pages 171-180, 2000.

[2] W. Aiello, F. Chung, and L. Lu. Experimental Math, 10:53, 2001.

[3] R. Albert and A.-L. Barabàsi. Topology of evolving networks: Local events and universality. Physical Review Letters, 85(4):5234-5237, 2000.

[4] R. Albert, H. Jeong, and A.-L. Barabàsi. Diameter of the world-wide web. Nature, 401:130-131, 1999.

[5] A.-L. Barabàsi and R. Albert. Emergence of scaling in random networks. Science, 286:509, 1999.

[6] A.-L. Barabàsi, R. Albert, and H. Jeong. Mean-field thory for scale-free random networks. Physica A, 272:173-187, 1999.

[7] A.-L. Barabàsi, R. Albert, and H. Jeong. Scale-free characteristics of random networks: The topology of the World Wide Web. Physica A, 281:69-77, 2000. 
[8] M. Boguná, R. Pastor-Satorras, and A. Vespignani. Cut-offs and finite size effects in scale-free networks. Eur. Phys. J. B, 38:205-209, 2004.

[9] C. Borgs, J. Chayes, M. Mahdian, and A. Saberi. Exploring the community structure of newsgroups. Conference on Knowledge Discovery in Data, Proceedings of the tenth ACM SIGKDD international conference on Knowledge discovery and data mining, pages 783-787, 2004.

[10] R. Breiger, S. Boorman, and P. Arabie. An algorithm for clustering relations data with applications to social network analysis and comparison with multidimentional scaling. Journal of Mathematical Psychology, 12:328-383, 1975.

[11] A. Broder, R. Kumar, R. Kumar, F. Maghoul, P. Raghavan, S. Rajagopalan, R. Stata, A. Tomkins, and J. Wiener. Graph structure in the Web. Computer Networks, 33:309, Jun 2000.

[12] A. Broida and K. Claffy. Internet topology: Connectivity of IP graphs. Scalability and Traffic Control in IP Networks in Proc. SPIE, International Society for Optical Engineering, (4526):172-187, 2001.

[13] M. Catanzaro, M. Boguná1, and R. Pastor-Satorras. Generation of uncorrelated random scale-free networks. Physical Review, 71:027103, 2005.

[14] H. Chang, R. Govindan, S. Jamin, S. J. Shenker, and W. Willinger. Towards Capturing Representative AS-level Internet Topologies. Computer Networks Journal, 44:737$755,2004$.

[15] H. Chang, S. Jamin, and W. Willinger. Inferring AS-level Internet Topology from Router-Level Path Traces. in Proc. of SPIE ITCom, 2001.

[16] Q. Chen, H. Chang, R. Govindan, S. Jamin, S. J. Shenker, and W. Willinger. The Origine of power laws in Internet topologies revisited. INFOCOM, 2002.

[17] L. Dall'Asta, I. Alvarez-Hamelin, A. Barrat, A. Vazquez, and A. Vespignani. Exploring networks with traceroute-like probes: theory and simulations. Theoretical Computer Science, 6:355, 2006.

[18] G. Davis, M. Yoo, and W. Baker. The small world of the American corporate elite, 1982-2001. Strategic Organization, 1:301-326, Aug 2003.

[19] S. Dorogovtsev and J. Mendes. Evolution of networks. Advances in Physics, 51:1079, 2002.

[20] H. Ebel, L.-I. Mielsch, and S. Bornholdt. Scale-free topology of e-mail networks. Physical Review E, 66:035103, 2002.

[21] P. Erdös and A. Rènyi. On random graphs I. Puble, Math, 6:290, 1959.

[22] M. Faloutsos, P. Faloutsos, and C. Faloutsos. On Power-Law Relationships of the Internet Topology. Computer Coтmunication Review, 29:251-262, 1999.

[23] R. FerreiCancho, C. Janssen, and R. Sole. Topology of technology graphs: Small World patterns in electronic circuits. Physics Review, 64:046119, 2001.

[24] M. Girvan and M. Newman. Community structure in social and biological networks. PROC.NATL.ACAD.SCI.USA, 99:7821, 2002

[25] J. Grossman and P. Ion. On a protion of the well-known collaboration graph. Congressus Numerantium, 108:129131,1995
[26] R. Guimerá, L. Díaz-Guilera, F. Giralt, and A. Arenas. Selfsimilar community structure in or ganisations. Preprint cond-mat/0211498, 2002.

[27] M. R. Horton. Standard for Interchange of USENET Messages, 1983. RFC850.

[28] H. Jeong, S. Mason, A.-L. Barabàsi, and Z. Oltvai. Lethality and centrality in protein networks. Nature, 411:41, 2001.

[29] B. Kantor and P. Lapsley. Network News Transfer Protocol, 1986. RFC977.

[30] B. Kernighan and S. Lin. An efficient heuristic procedure for partitioning graphs. Technical Report, Bell Systems Technical Journal, 49(2):291-307, 1970.

[31] J. Kleinberg, R. Kumar, P. Raghavan, S. Rajagopalan, and A. Tomkins. The Web as a graph. Proc. 19th ACM SIGACTSIGMOD-AIGART Symp. Principles of Database Systems, PODS, ACM Press, pages 1-10, Jun 1999.

[32] N. Martinez. Artifacts or Attributes? Effects of Resolution on the Little Rock Lake Food Web. Ecological Monographs, 61:367, 1991.

[33] M. Newman. The structure of scientific collaboration networks. PROC.NATL.ACAD.SCI.USA, 98:404, 2001.

[34] M. Newman. Assortative mixing in networks. Physical Review Letters, 89:208701, 2002.

[35] M. Newman. Fast algorithm for detecting community structure in networks. Physical Review E, 69:066133, 2004.

[36] M. Newman, S. Forrest, and J. Balthrop. Email networks and the spread of computer viruses. Physical Review E, 66:035101, 2002.

[37] M. Newman and M. Girvan. Finding and evaluating community structure in networks. Physical Review E, 69:026113, 2004.

[38] M. Ripeanu, I. Foster, and A. Iamnitchi. Mapping the Gnutella network: Properties of large-scale peer-to-peer systems and implications for system design. IEEE Internet Computing, 6:50-57, 2002.

[39] Y. Saito, J. Mogul, and B. Verghese. A Usenet Performance Study. 1998. http://www.research.digital.com/wrl/projects/ newsbench/usenet.ps.

[40] J. Scott. Social Network Analysis, 2nd ed. A Handbook, Sage, Publications, London, 2000.

[41] G. Siganos, M. Faloutsos, P. Faloutsos, and C. Faloutsos. Power laws and the AS-level internet topology. IEEE/ACM Trans. Netw., 11(4):514-524, 2003.

[42] E. Sit, F. Dabek, and J. Robertson. UsenetDHT: A low overhead usenet server. In In Proc. of the 3rd IPTPS, Feb 2004.

[43] R. Smith. Instant messaging as a scale-free network. Preprint cond-mat/0206378, 2002.

[44] TOP1000org. The Official Usenet TOP1000 Servers page. http://www.top1000.org/.

[45] A. Vázquez. Growing network with local rules: Preferential attachment, clustering hierarchy, and degree correlations. Phys. Rev. E, 67:056104, 2003.

[46] D. Watts and S. Strogatz. Collective dynamics of smallworld networks. Nature, 393:440, 1998.

[47] J. Winick and S. Jamin. Inet3.0: Internet Topology Generator. Tech Report UM-CSE-TR-456-02, 2002. 